periphere Veranlassung. Die Differenz ist zu gering, um zu gunsten der Jacquetschen Hypothese zu sprechen.

Ward, E. Rudimentäre Marsupialtasche beim Menschen. p. 366 .

Ward beobachtete bei einem sonst gesunden Knaben folgende Anomalie: In der Mittellinie fand sich über dem Processus xiphoideus sterni eine Hauttasche, in die eine kleine öffnung linkerseits führte. Die Tasche war innen von glatter, normaler Haut ausgekleidet. An der Haut der Tasche fanden sich keine Zeichen von Narbenbildung. Der Verfasser nimmt an, daß es sich um eine rudimentäre Marsupialtasche handelt, da sie auch dort lokalisiert ist, wo der Beutel bei den Marsupialiern sitzt.

Fritz Juli usberg (Posen).

\title{
The Journal of cut. diseases includ. Syph.
}

XXX. August 1912. Nr. 8.

Hartzell. Philadelphia. Erythema ab ig ne. p. 461 .

Die dermatologische Literatur bringt nur wenig über das durch lange Hitzeapplikation hervorgerufene netzförmige Erythem und die entsprechende Pigmentablagerung. Hartzell hat vier derartige Fälle gesehen, bei denen zumeist an den unteren Extremitäten, bei einem an der Lumbosakralgegend ausgedehnte flächenförmige Herde netzförmiger Pigmentierung vorhanden waren, welchen laut Anamnese ebenso geformte Erytheme vorausgegangen waren. Als Ursache konnte bei allen entweder direkte Feuer-(Ofen-) Bestrahlung aus der Nähe oder die langdauernde Applikation von Wärmefaschen nachgewiesen werden. Histologisch fand er bei einem Fall (langdauernde Wärmeflaschenapplikation) Parakeratose und Verbreiterung des Rete; bei einem zweiten Mangel des Stratum granulosum und Exsudation ins papillare Stratum, sowie in dem Fall von Erythem (I) Exsudation um die Schweißdrüsen. Alle Schnitte zeigten eine starke und deutlich abgegrenzte Ablagerung von braunem, körnigem Pigment in der basalen Schleimschichte. Bei allen seinen Fällen konnte Hartzell Gesundheitsschädigungen, wie Alkoholismus, Alter, überstandene Krankheit beobachten und er zweifelt, daß die Anomalie bei völlig Gesunden vorkommt.

In der Diskussion stimmen $Z$ eisler und G. H. Flox der durch Hartzell gegebenen Krankheitsschilderung bei und möchten nur in der Nomenklatur statt des Feuers "Wärme" wählen: Erythema ab calore. 
Fox, Howard. New-York. Ein. Versu ch mit der Luetinreaktion von Noguchi. p. 465.

Fox hat eine Prüfung der Luetinreaktion Noguchis Injektion von durch Hitze abgetöteten Spirochaetereinkulturen - bei Luetischen vorgenommen und ist zu im ganzen bemerkenswerten Resultaten gelangt. 1/10 $\mathrm{ccm}$ des Luetin wurde möglichst oberflächlich in die Haut eines Armes eingespritzt, auf die andere Seite die gleiche Menge des nicht infizierten Nährmediums als Kontrolle. In einigen Fällen war etwas Kopfschmerz und Übelbefinden zu beobachten, die lokalen Beschwerden waren meist gering. Die positive Reaktion begann am Abend (nach der Injektion) und war nach 48-72 Stunden auf ihrer Höhe; sie bestand in einem tief sitzenden entzündlichen Knötchen mit einem verschieden großen roten Hof, in mehreren Fällen traten Pusteln auf. Bei den nicht syphilitischen Kontrollfällen war manchmal eine schwache, aber nie eine ausgesprochene Lokalisation vorhanden.

In 14 Fällen akquirierter Lues war die Luetinreaktion in 6, die Wassermannreaktion in 13 Fällen positiv; in 33 Fällen tertiärer und latenter Syphilis war die Luetinreaktion 17 mal, die $W$ assermannreaktion $19 \mathrm{mal}$ positiv.

5 gut mit $\mathrm{Hg}$ behandelte, tertiäre und lokale Fälle gaben 1 positive und 4 negative Luetinreaktion. 11 mit einer oder mehreren Salvarsaninjektionen behandelte hatten 3 positive und 8 negative Luetinreaktionen.

In 10 Fällen war die Reaktion auf beiden Armen fast gleich (! ?).

Die meisten positiven Reaktionen hinterließen einen bläulichen Fleck oder winzige Keloide.

Diskussion: Auch Prsey bestätigt die merkwürdige Tatsache, daß bei ausgesprochen positiver Reaktion die Kontrollstelle gleichfalls positiv reagierte; umgekehrt verhielt sich die negative. Wassermann korrespondierte im allgemeinen namentlich bei Spätsyphilis, weniger bei der Frühsyphilis mit der Luetinreaktion.

Engman findet, daß die Kontrollreaktion (auf dem 2. Arm) nicht so ausgesprochen sei, wie jene auf dem ersten.

W infield hat die Luetinreaktion verfolgt. In 14 Fällen von Nervenlues war $W$ assermann 9 mal negativ, 1 mal positiv; Noguchis Reaktiın 10 mal positiv. In 3 Fällen spastischer Paraplegie Wa s s rman jedesmal negativ, Noguchi positiv; von 6 hereditär-luetischen Fällen 3 positiv Wassermann, 4 positiv Noguchi. Von den Luesfällen mit sichtbaren Erscheinungen überwog in den Sekundärfällen der positive Ausfall des Wassermann jenen des Noguchi, dagegen war No$\mathrm{guch} \mathrm{i}$ in den Tertiärfällen häufiger positiv.

Pollitzer findet, daß nar ein geringer Teil der Sekundärfälle positiv nach Noguchi reagiert, von den Tertiärfällen etwas über $50 \%$. Weiters ist es unangenehm, daß schwache Noguchireaktionen auch bei Nichtlnetischen erzielt wurden. Vielleicht liegt der Wert der Reaktion, wenn sie deutlich positiv ausfällt, in den dunklen Luesfällen, bei denen Wassermann negativ reagiert. 
Sutton, Richard L. Kansas City. Die Alopekien im allgemeinen, mit besonderer Berücksichtigung einer bisher nicht beschriebenen Form von Kahlheit: der "Alopecia indurata atrophica“. p. 471 .

Sutton gibt zuerst eine Zusammenstellung der verschiedenen Alopekien, wobei er ein von Brocq aufgestelltes System etwas modifiziert. Die Alopekien zerfallen hiernach in solche, die wirkliche Krankheitseinheiten darstellen und in solche, welche die Folge einer Hautreaktion sind. Zu letzteren zählt er die auf Nervenalteration bezogenen. Er berichtet anschließend über drei klinisch und histologisch merkwürdige Fälle.

Der erste Fall betraf eine früher stets gesunde 39jährige Frau, bei der die Haare seit 6 Jahren kürzer und dünner wurden und bei jedem Kämmen massenhaft mit der Haarwurzel ausgingen oder im Follikel abbrachen. Neben Kürze und Spärlichkeit der Haare war die Rauheit und straffe Anheftung der Kopfhaut bemerkenswert.

Auch beim 2. Falle, einer 27jährigen Lehrerin, war ohne Veranlassung vor 7 Jahren Verdünnung, ungleichmäßige Färbung und auffallend starker Haarveriust aufgetreten. Sie bekam das Gefühl, als würde ihr ein Band um den Schädel gezogen. Die Haare wurden hart, trocken, brüchig, die Haut dünn und fest angezogen, fast unbeweglich. Histologisch war eine bedeu. tende Verdünnung aller Epidermisschichten, Abflachung des Papillarkörpers, Verdichtung und Degeneration des Bindegewebes und völliger Mangel des subkutanen Fettes nachweisbar. Die Haarfollikel waren bedeutend zusammengeschrumpft und atrophisch, mehr als die Talgdrüsen. Kapillaren des oberen Drittels des Dermas in Größe und Zahl deutlich vermindert.

Im 3. Fall ist klinisch neben Verdünnung und Ausfall der Haare, starker Verdünnnng und Spannung der Kopfhaut, noch das Auftreten von Neuralgien bemerkenswert, welche abwechselnd verschiedene Kopfregionen befielen. Histologisch ähnliche, aber stärkere Veränderungen wie im Falle 2. Therapeutisch war bei allen Fällen wenig zu erreichen, am wirkungsvollsten noch die Massage.

Sutton hält diese Alopecia indurata atrophica, welche dem Skleroderma am nächsten steht, für eine neurotische Affektion (in Gruppe 6 Brocq).

Keyes, E. J. Ir. Tod nach Gebrauch von Salvarsan. Brief an den Herausgeber. p. 478.

Der Patient von Ke yes, ein großer athletischer Mann, hatte wegen eines papulösen Syphilids (Geschwüre an den stark vergrößerten Tonsillen) Salvarsan $0 * 4$ alkalisch intravenös erhalten, was er ohne unmittelbare Reaktion vertrug. Doch be- 
kam er bald darauf hohes Fieber, Tonsillenschwellung und gelbe Konjunktiva.

18 Tage nach der ersten erhielt er von $\mathrm{K}$. eine Injektion von 0.3 Salvarsan in saurer Lösung, bekam darauf Kongestionen, wurde schwach und ohnmächtig, bekam Erbrechen, Diarrhoen, fliegenden und schwachen Puls und wurde unbesinnlich. Später stellte sich Erythema toxicum, Ikterus, Albuminurie und Retentio urinae ein und Patient ging nach einem Krankenlager von 7 Tagen zu grunde. Verf. glaubt nicht, daß der sauren Lösung noch der poliklinischen Verabreichung des Mittels eine Schuld beizulegen ist. Doch hält er für unbedingt nötig, daß die Injizierten nachher nicht ohne ärztliche Pflege bleiben, was in dem berichteten Fall zu Beginne nicht geschehen war.

September 1912. Nr. 9.

Moore, George D. Ph. D. Krebs bei Pflanzen. (Einges. v. Herausgeber.) p. 515.

Erst in jüngster Zeit ist es den Pflanzenpathologen gelungen, ähnliche Bedingungen für das Wachstum abnormer Geschwülste der Pflanzen, wie sie beim menschlichen Krebs vorhanden sind, zu zeigen. Die sogenannte Kronengalle, seit 50 Jahren bekannt, erzeugt bei Rosen, Himbeeren, Trauben und rerschiedenen Fruchtbäumen Störungen, zu deren Erklärung dieselben Momente wie für das menschliche Karzinom herangezogen wurden. Bei der Kronengalle ist nicht nur äußerliche Vergrößerung wie bei den meisten Pflanzentumoren vorhanden, sondern eine echte Hyperplasie. Die Zelle und ihre enorme Vermehrung ist das Element der physiologisch unbegründeten Vermehrung. Noch interessanter sind die entfernten (metastatischen) Pflanzentumoren, welche Tendenz zur Entwicklung des primären Tumorgewebes zeigen. Spontane Heilung sowie operative durch komplette Entfernung der Tumoren sind nicht selten, bei manchen gibt es eine erworbene Immunität. Zum Unterschied vom Karzinom ist aber die Ursache der Krongalle bekannt: das isolierbare Bacterium tumefaciens. Seine Entdeckung brauchte wegen der geringen Anzahl in den Tumoren, der schweren Anreicherung und Kultivierung außerhalb derselben und der schweren Einimpfbarkeit und Färbbarkeit lange Zeit. Auf Kaltblüter geimpft erzeugt es dem Karzinom äbnliche Knötchen im Bindegewebe, eine jedenfalls merkwürdige und erwägungsreiche Tatsache.

Pernet, George, London, England. Dermatitis pustulosa vegetans recurrens. p. 517.

Sieben Monate nach einer mäßig schweren (Zangen-)Geburt traten bei einer Frau am Kopfe und nn der Nase kleine weißliche Knoten auf, die eine helle Flüssigkeit absonderten. Später 
wurde die Absonderung eitrig, die Knötchen wurden Blattern, die Vulva und Innenseiten der Oberschenkel wurden ergriffen und es trat eine Kolitis eín. Bei der nach mehrfachen Besserungen und Rückfällen erfolgten Spitalsaufnahme war ein großer Teil des Kopfes, des Nackens, die Obren, die Nase mit dicken Krusten versehen, verdickt; am Rande Kreise und Kreissegmente von Pusteln aufweisend. An den glatten Partien sind die Effloreszenzen erhaben, die Stirnfalten sind deutlicher ausgeprägt. Die Pusteln gewisser Stellen bilden sezernierende, vegetierende Flächen. Die Nase nur am Eingang ergriffen, vom Rücken der größte Teil bis zum Gürtel, der Thorax voru bis zur Mamillarhorizontalen, die Regio pubis und einzelne andere $\cdot$ Körperstellen.

Die Affektion hatte Ähnlichkeit mit von Hallopeau (Derm. pust. chron. en foy. à progr. excentr.), Hebra (Impetigo herpetiformis), A uspitz (Herpes vegetans), $\mathrm{Neumann}$ (Herpes pyaemicus) beschriebenen Fällen, doch möchte Pe r n e t, ohne die differenziellen Momente erschöpfend auszufübren, ihn für verschieden von jenen halten. Ätiologisch ist er ungeklärt. Sowohl äußere Therapie (Jodoformsalbe) als Vakzinebehandlung (autogene Staphylokokkenvakzins) hatten zeitweilige Besserungen zur Folge, doch traten letztere auch spontan ein; ebenso häufig rezidivierte die Affektion.

Montgomery, Douglass W. und Culver, George D., San Franzisco. Tricbloressigsäure als keratolytisches Mittel bei seborrhoischer Keratose. p. 523.

Herde seborrhoischer Keratose ev. praecanceröse Herde werden durch Trichloressigsäure besser als durch X-Strahlen, Kohlensäure, Elektrolyse und Fulguration beeinflußt.

Nur muß die Handhabung zweckmäßig sein. Die Trichloressigsäure muß in Kristallen (Vorsicht auf die Augen!) oder in konzentrierter wässeriger Lösung mit Wattebäuschchen auf getragen, daselbst bis zu deutlicher Weißfärbung appliziert erhalten und dann sofort mit Wasser sorgsam abgetupft werden, bis jeder Schmerz aufhört. Sie macht je nach der Tiefe ihrer Anwendung keine Narben, oder wenn das Bindegewebe erreicht wird, Narben. Sie scheint eine gewisse Affinität zum Fett zu haben, woher möglicherweise ihre gute Wirkung bei seborrhoischen Produkten, wie sie Eisessig zum Horngewebe hat, daher die beliebte Anwendung des letzteren bei Hühneraugen.

Mac Kee, George M. und Remer, John, New-York, Di e Einzeldosen X-Bestrablungsmethode. p. 528.

Die Methode der vereinzelten Röntgenstrahlenapplikation setzt eine genaue Kenntnis in der Verwendung der Qualität (weich, mittelweich, hart) und der Quantität der benutzten Strahlen voraus. Die erstere wird, aber nur für die stärkst 
penetrierenden Strablen, mit dem Be no is t schen Radiochromometer gemessen, braucht aber wiederholte Kontrolle. Die zweite wird gegenwärtig mit den Pastillen von Sabouraud-Noiré und dem Radiometer von Holzknecht bestimmt. Mac Kee hat die Methode von Holzknecht modifiziert. Er verwendet mittelweiche Röhren, verringert die Entfernung von Haut- resp. Reagenspastille gegenüber dem Fokus und ändert die Bezeichnungen für die Strahlenquantität, indem für seine Technik $1 \mathrm{H}(\mathrm{Holzknecht}$ ) gleich ist $4 \mathrm{H}$ der Originalskala. Haut und Reagenskörper umgibt or mit Blenden, die Licht und Hitze abhalten müssen.

Der Vorteil der Eindosismethode ist der einer bedeutend rascheren Wirkung. Ein Haarausfall am ganzen Schädel z. B. ist in 16-25 Minuten erhältlich. Sykosis heile im allgemeinen auf eine einzige Dosis. Epitheliome sollen grändlicher resp. ohne die bei der alten Methode häufigen Rezidiven heilen, da bei der letzteren das Hautgewebe durch die wiederholten milden Bestrahlungen gereizt eine Art Immunisierung gegen die Röntgenstrahlen erworben hatten. Dagegen hat die Eindosismethode auch dort keinen Nutzen, wo Röntgenstrahlen überhaupt wirkungslos sich gezeigt haben.

Diskussion: Pusey bestreitet den Vorzug der Einzeldosismethode, ihr stehe namentlich die verschiedene Empfindlichkeit der Leute gegenüber den Strahlen entgegen. Er habe die besten Resultate mit fraktionierten Dosen auch bei Epitheliomen gesehen, und würde heroische Dosen auf große Flächen wie z. B. bei Leukämie nicht wagen. Hauptbedingung für gute Resultate sei die persönliche, ersthandige Vertrautheit mit der Technik.

Zeisler hält die von Mac Kee mitgeteilte Technik für den dermatologischen Praktiker für schwer und nur für spezialistisch geschulte Leute geeignet.

Bulkley berichtet dagegen über die vorzüglichen Resultate, die er bei Colcott Fox in London und bei Sabouraud in Paris mit der Eindosismethode bei Kopffavus and Herpes tonsurans gesehen. Rezidiven waren äußerst selten und Dermatitiden keine. Freilich hat eine Wiederholung der Methode in Amerika, vielleicht wegen der sohlechten Qualität der Reagenspastillen, nicht dieselben guten Resultate gegeben.

Mac Kee resümiert, daß die neue Methode wissenschaftlich und genau, die alte nur eine ungefäbre sei. Um genaue und prompte Resultate zu erzielen, muß freilich die Technik vollendet und Vorsicht, Verstand und Wachsamkeit angewendet werden.

Rudolf Winternitz (Prag).

\section{Annales de Dermatologie et de Syphiligraphie.}

1912. Nr. 8 und 9.

Balzer, Gougerot und Burnier. Über eine dur ch das Mycoderma pulmoneum verursachte disseminierte wachernde Dermatomykose. p. 461. 